\title{
A Navigation and Examination Aid for 3D Virtual Buildings
}

\author{
Luca Chittaro, Vijay Kumar Gatla, and Subramanian Venkataraman \\ HCI Lab, Dept. of Math and Computer Science, University of Udine, \\ Via delle Scienze 206, 33100, Udine, Italy \\ \{chittaro, gatla, venkatar\}adimi.uniud.it
}

\begin{abstract}
In this paper, we present the Interactive 3D BreakAway Map (I3BAM), an extension of Worlds In Miniature (WIM) that works not only as a navigation aid for virtual buildings but also provides a means of examining any floor of a virtual building without having to navigate it.
\end{abstract}

\section{Introduction}

In several virtual environment (VE) applications, users navigate 3D buildings. Typical examples can be virtual shopping malls, virtual museums, 3D games, simulations where users learn routes and building structure to be applied in real life scenarios [1], virtual models of complex real buildings where structural changes to rectify design defects are not feasible, thus creating the need for alternative solutions [2]. This paper presents the I3BAM, an extension of WIM that works not only as a navigation aid for virtual buildings but also provides a means of examining any floor without having to navigate it.

\section{Related Work and Motivations}

Several aids have been proposed in the literature to provide navigation support in VEs (e.g., $[3,5,6,7])$. Buildings are one of the most common types of VEs. Navigation within a virtual building (to learn a route to a particular location or gain structural knowledge) is often not the only task and the user's cognitive resources can be needed for additional tasks (e.g., object manipulation, collecting information etc.). The WIM is a well-known metaphor that uses 3D miniature maps to provide the user with object manipulation and locomotion capabilities [5]. A WIM is a 3D miniature version of the $\mathrm{VE}$, floating in front of the user, as if it were in her virtual hand. User's position and orientation are indicated in the WIM. Although intuitive, the WIM has shortcomings especially related to navigation [5,6], e.g., merely presenting a WIM of an entire building would not provide a means of studying both the external and internal structure of the building (floor wise), the importance of which has been stressed in building structure visualization [4]. No previous attempts have been made to apply the WIM to virtual buildings (except for a prop based technique [7]). The I3BAM extends the WIM and applies it to virtual buildings aiming at providing both: i) a navigation 
aid - for users who travel in the VE by walking; ii) a means of studying any floor without having to navigate it.

\section{The Interactive 3D BreakAway Map}

For designing the I3BAM we aimed at providing: i) easy to understand information to help the user gain route and survey knowledge of a building; ii) easy interaction; iii) the capability of studying the structure of any floor without having to navigate it; iv) multiple views of the building, including a view of user's immediate surroundings with respect to the global context; v) enhancements to the user's exploration and discovery process.

The I3BAM provides the user with knowledge about the external and overall structure (e.g., the number of floors, exits,...) and also the internal structure of the building (e.g., how the floors are connected by staircases, where staircases are located, structural details of each floor,...). The I3BAM is a miniature 3D model of the building that constantly floats in front of the user. It can be moved and resized to avoid unwanted occlusions and it can be flipped to gain the best (ranging from a front to a top down) view of the building, by means of slider controls (Fig 1). Three different views (described in the following) are provided by the I3BAM, each one presenting the building at varying amounts of visual complexity. Operating on the views does not affect the virtual building in any manner.

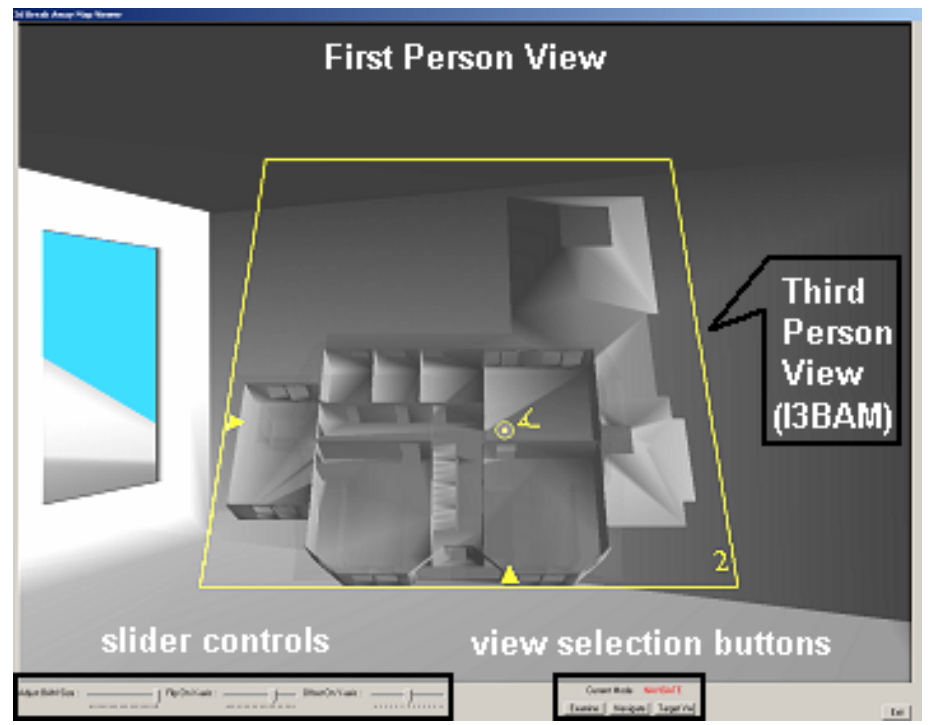

Fig. 1. The I3BAM (center of the screen) with the related controls (bottom of the screen)

Examine: In this view, the user can interact with the I3BAM via mouse clicks. All floors are visualized. Each floor slides out (horizontally) when clicked. This allows the user to move floors obstructing the view to a particular floor and study its 
structure. It also helps to understand how the floors are organized to form the complete building. The floor sliding action is toggle-controlled: one click slides a floor outward from its initial position, another click slides it back to the initial position, e.g. being on floor 1 (below the white rectangle in Fig 2 and 3), the user can examine the structure of other floors: she clicks on floor 4 which slides out in the direction of the arrow (Fig 2) revealing the structure of floor 3, then she clicks on floor 3 to reveal floor 2 (Fig 3).

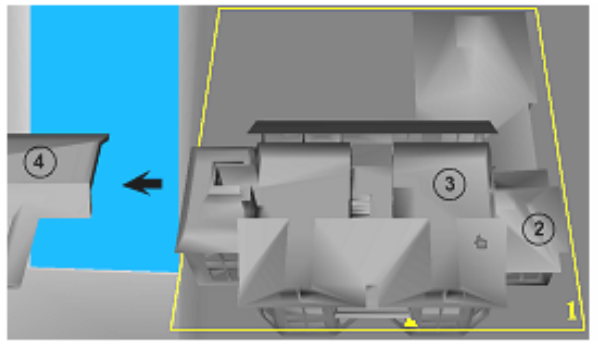

Fig. 2. Examine View (before click on floor 3)

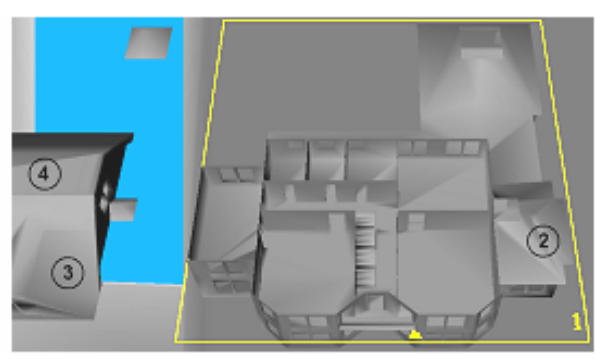

Fig. 3. Examine View (after click on floor 3)

Floor: In this view, the I3BAM displays mainly the floor on which the user is walking. The rest of the building becomes semi-transparent, making it possible to use the view as a 3D map to navigate the current floor. In Fig 4, the I3BAM shows floor 1 (below the white rectangle). The white colored glyph indicates user's current position and orientation. The two white arrowheads along the sides are user's position along the length and breadth of the floor.

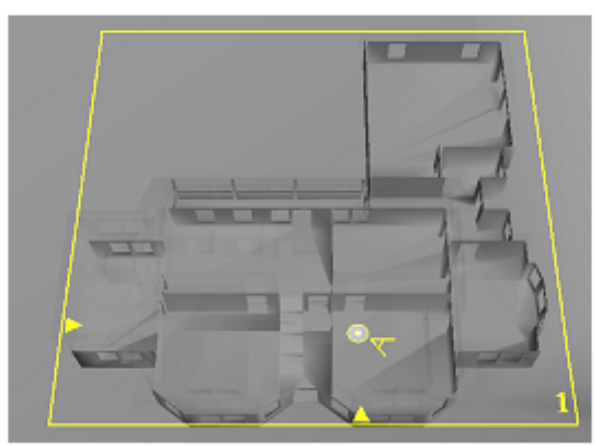

Fig. 4. Floor View

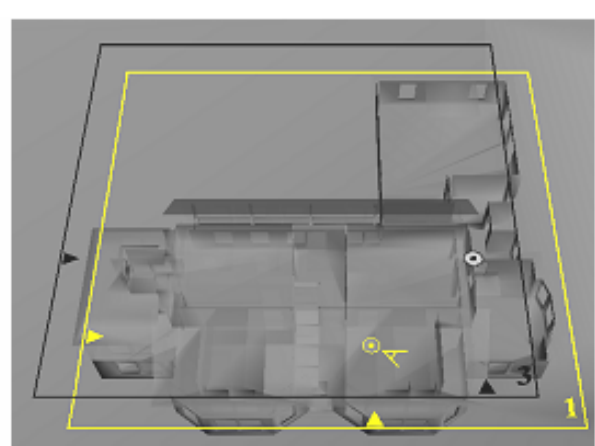

Fig. 5. Target View

Target: In this view, the I3BAM simultaneously displays the position of the user and a selected target within the global context of the entire building, enabling the user to assess her own position with respect to the target and decide how to reach it, by navigating. In Fig 5, the I3BAM shows the user's glyph is on floor 1. The target floor is outlined by the black rectangle and the target position by the black and white spot. The arrowheads (white for user's position and black for target position) further help 
the user to reach the target: once on the target floor, the user moves towards the target position until both the white and black arrow pairs coincide. The transparencies of floors are varied to provide the best possible view to the user. Let us call 'Higher' as the higher among the user and target floors and the other one as 'Lower'. All floors below the Lower floor are completely opaque, those between the Lower and Upper floor are rendered $90 \%$ transparent. The Higher floor is rendered 50\% transparent. All floors above the Higher floor are rendered completely transparent.

The three views are easily differentiated visually and the name of the active view is also shown in text. Transitions among views are animated. Route knowledge can be gained by using the I3BAM because the user is made aware of the possible directions she can take and her own bearings with respect to the target as she travels towards it. Furthermore, survey knowledge can be gained both by studying (e.g., using the examine view) and actively exploring the building.

\section{Conclusions and Future Work}

The I3BAM extends the WIM (and attempts to overcome some of its shortcomings), applying it to virtual buildings both as a navigation aid and as a tool to study the building. We are now evaluating it on users and preliminary results are promising. We are also currently working on the development of tools to apply the I3BAM to previously created building models and on the integration of a path planning algorithm to provide detailed route indication capabilities. Finally, we plan to carry out a training transfer study based on routes to offices in our university building.

\section{References}

1. Bliss, J.P., Tidwell, P.D, Guest, M. The effectiveness of virtual reality for administering spatial navigation training to firefighters. Presence: Teleoperators and Virtual Environments 6, 1, pp.73-86, 1997.

2. Kalkusch, M., Lidy, T., Knapp, M., Reitmayr, G.,Kaufmann, H.,Schmalstieg, D. Structured visual markers for indoor pathfinding. Proc. of 1st IEEE International Augmented Reality Toolkit Workshop, IEEE Press, 2002.

3. Ruddle, R.A., Payne, S.J., Jones, D.M. The effects of maps on navigation and search strategies in very-large-scale virtual environments. Journal Of Experimental Psychology: Applied 5, pp.54-75, 1999.

4. Niederauer, C., Houston, M., Agrawala, M., Humphreys, G. Non-invasive interactive visualization of dynamic architectural environments. Proc. of the 2003 Symposium on Interactive 3D Graphics, ACM Press, New York, pp.55-58, 2003.

5. Stoakley, R., Conway, M., Pausch, R. Virtual reality on a WIM: Interactive worlds in miniature. Proc. of CHI95, ACM Press, New York, pp.265-272, 1995.

6. Pausch, R., Burnette, T., Brockway, D., Weiblen, M.E. Navigation and Locomotion in virtual worlds via flight into hand held miniatures, Proc. of SIGGRAPH '95, ACM Press, New York, pp.399-400, 1995.

7. Brooks, A. Aids for training real-world spatial knowledge using virtual environments. In Thompson B.J. (ed.) Research Papers of the Link Foundation Fellows (3), University of Rochester Press, 2003.

8. Murphy, M. "Home 07”, (Public Domain) Building Model, www.pacranch.com 